\title{
三甲基膦支持的硒配位钴氢配合物的合成和性质
}

\author{
奕化金金孙宏建薛本静李晓燕* \\ (山东大学化学与化工学院 教育部特种功能聚集体材料重点实验室 济南 250199)
}

\begin{abstract}
摘要 通过 4-甲氧基苯硒酚和 2-甲基苯硒酚分别与 $\mathrm{Co}\left(\mathrm{PMe}_{3}\right)_{4}$ 反应生成了单核 $\mathrm{Co}(\mathrm{II})$ 配合物 $\left[\mathrm{Co}\left(4-\mathrm{MeOC}_{6} \mathrm{H}_{4} \mathrm{Se}\right)_{2}\right.$ $\left.\left(\mathrm{PMe}_{3}\right)_{3}\right](\mathbf{1})$ 和双核 $\mathrm{Co}(\mathrm{I})$ 配合物 $\left[\mathrm{Co}\left(\mathrm{PMe}_{3}\right)_{2}\left(\mathrm{SeC}_{6} \mathrm{H}_{4}-2-\mathrm{Me}\right)\right]_{2}$ (2). 配合物 2 与 $\mathrm{CO}$ 作用形成了配合物 $\left[\mathrm{Co}\left(\mathrm{PMe}_{3}\right)_{2}(\mathrm{CO})_{2}(\mathrm{Se}-\right.$ $\left.\left.\mathrm{C}_{6} \mathrm{H}_{4}-2-\mathrm{Me}\right)\right](3)$. 而苯硒酚与 $\mathrm{Co}\left(\mathrm{PMe}_{3}\right)_{4}$ 反应制备了硒配位的钴氢配合物 $\left[m e r-\mathrm{Co}(\mathrm{H})(\mathrm{SePh})_{2}\left(\mathrm{PMe}_{3}\right)_{3}\right](4)$ 和双核 $\mathrm{Co}(\mathrm{I})$ 配 合物 $\left[\mathrm{Co}(\mathrm{PMe})_{2}(\mathrm{SePh})\right]_{2}(\mathbf{5})$. 初步研究表明 4 对于醛和酮的硅氢化反应有一定的催化效果. 配合物 1, 3 和 4 的分子结构 通过 $X$ 射线衍射进行了证实.
\end{abstract}

关键词 钴氢配合物; 硒酚; 硒配位; 硅氢化反应; 羊基钴配合物

\section{Synthesis and Properties of a Se-Coordinated Hydrido Cobalt(III) Complex Supported by Trimethylphosphine}

\author{
Luan, Huaxin Sun, Hongjian Xue, Benjing Li, Xiaoyan* \\ (Key Laboratory of Special Functional Aggregated Materials, Ministry of Education, School of Chemistry and \\ Chemical Engineering, Shandong University, Jinan 250199)
}

\begin{abstract}
A mononuclear cobalt(II) complex $\left[\mathrm{Co}\left(4-\mathrm{MeOC}_{6} \mathrm{H}_{4} \mathrm{Se}\right)_{2}\left(\mathrm{PMe}_{3}\right)_{3}\right]$ (1) and a dinuclear cobalt diphenylselenolato complex $\left[\mathrm{Co}\left(\mathrm{PMe}_{3}\right)_{2}\left(\mathrm{SeC}_{6} \mathrm{H}_{4}-2-\mathrm{Me}\right)\right]_{2}$ (2) were obtained from the reaction of 4-methoxyselenophenol and 2-methylselenophenol with $\mathrm{Co}\left(\mathrm{PMe}_{3}\right)_{4}$, respectively. The dicarbonyl cobalt(I) complex $\left[\mathrm{Co}\left(\mathrm{PMe}_{3}\right)_{2}(\mathrm{CO})_{2}\left(\mathrm{SeC}_{6} \mathrm{H}_{4}-2-\mathrm{Me}\right)\right](3)$ was formed through the reaction of complex $\mathbf{2}$ with carbon monoxide. A novel selenophenolato hydridocobalt(III) complex $\left[\right.$ mer- $\left.\mathrm{Co}(\mathrm{H})(\mathrm{SePh})_{2}\left(\mathrm{PMe}_{3}\right)_{3}\right](4)$ and a dinuclear cobalt diphenylselenolato complex $\left[\mathrm{Co}\left(\mathrm{PMe}_{3}\right)_{2}(\mathrm{SePh})\right]_{2}(\mathbf{5})$ were synthesized by the reaction of selenophenol with $\mathrm{Co}\left(\mathrm{PMe}_{3}\right)_{4}$. It was found that complex 4 could catalyze the hydrosilylation of aldehydes and ketones. The molecular structures of $\mathbf{1 , 3}$ and $\mathbf{4}$ were determined by X-ray diffraction.

Keywords hydrido cobalt complex; selenophenol; Se-coordinated; hydrosilylation; carbonyl cobalt complex
\end{abstract}

过渡金属氢化物是一类重要的金属有机化合物，被 广泛应用于催化化学、合成化学和化学工业过程等各个 领域. 人们已经制备了一系列的过渡金属(尤其是贵金 属)的氢化物, 并且研究了这些金属氢化物的催化作 用 ${ }^{[131]}$. 富电性金属铼的氢化物可以催化烯烃的加氢反 应 $^{[8]}$. 傅尧等 ${ }^{[9]}$ 在 2015 年利用 Casey 的铁氢催化剂实现 了乙酰丙酸乙酯的转移氢化反应, 并最终得到 $\gamma$-戊内 酯. 刘湘和李和兴等利用 $\mathrm{Cu}(\mathrm{OAc})_{2} \cdot \mathrm{H}_{2} \mathrm{O}$ 为催化剂, 聚 甲基氢硅氧烷(PMHS)为氢源, 在手性膦配体 $(R)$-BINAP 存在下实现了芳香酮的不对称硅氢化反应 ${ }^{[10]}$. Milstein 等 ${ }^{[13]}$ 前不久报道了钉的 $[\mathrm{PNN}]$ 钳式氢化物可以有效地催 化碱性水溶液中甲醇制氢的转化, 作为副产物的 $\mathrm{CO}_{2}$ 可
以利用碱液来吸收. Haenel, Kaska 和 Hall 等 ${ }^{[23]}$ 披露了铱 的 $[\mathrm{PCP}]$ 钳式氢化物在均相催化条件下可以催化烷烃的 脱氢反应. 铑的氢化物在水溶液中既能与 $\mathrm{CO}$ 发生插入 反应, 也能与醛和烯烃进行加成反应 ${ }^{[30]}$. 相比较而言, 关于钴的金属氢化物的报道则比较少见 ${ }^{[32 \sim 41]}$. Linehan 等 ${ }^{[37]}$ 在 2014 年发现了 $\mathrm{Co}(\mathrm{dmpe})_{2} \mathrm{H}$ 配合物在十分温和的 条件下 $\left(101 \mathrm{kPa}, 21{ }^{\circ} \mathrm{C}\right)$ 可以实现 $\mathrm{CO}_{2}$ 的氢化, 产物为甲 酸. Chirik 等 ${ }^{[38}$ 发现钳式配体 $[\mathrm{CNC}]$ 的钴氢配合物可以 催化烯烃的加氢反应. 我们 ${ }^{[39]}$ 曾经以硫代水杨醛为预 配体合成了 $\mathrm{Co}(\mathrm{III})$ 的氢化物, 首次研究了该配合物对于 醛和酮中羰基的硅氢化反应的催化作用. 我们还利用双 $\mathrm{C}-\mathrm{H}$ 键的活化合成了 $[\mathrm{CNC}]$ 钳式 $\mathrm{Co}(\mathrm{III})$ 氢配合物, 该

\footnotetext{
* Corresponding author. E-mail: xli63@sdu.edu.cn

Received February 28, 2017; revised April 14, 2017; published online May 10, 2017.

Project supported by the National Natural Science Foundation of China (No. 21372143).

国家自然科学基金(No. 21372143)资助项目.
} 
配合物可以催化羰基化合物还原为醇的反应, 氢源为 $(\mathrm{EtO})_{3} \mathrm{SiH}^{[40]}$. 另外, 我们 ${ }^{[41]}$ 通过 $\mathrm{Si}-\mathrm{H}$ 键的活化合成了 $[\mathrm{PSiP}]$ 钳式 $\mathrm{Co}(\mathrm{III})$ 的氢化物, 实验证明该氢化物可以在 较温和条件下有效地催化 Kumada 偶联反应.

在早期的工作中, 我们 ${ }^{[42]}$ 利用苯硫酚与 $\mathrm{CoCl}-$ $\left(\mathrm{PMe}_{3}\right)_{3}$ 或者与 $\mathrm{Co}\left(\mathrm{PMe}_{3}\right)_{4}$ 的反应制得了硫原子配位的 钴氢配合物, 并且研究了钴氢配合物的化学性质. 硒和 硫作为同一主族的元素, 尽管化学性质上具有一定的相 似性, 作为不同周期的化学元素, 化学性质的差异性还 是很明显的. 为了拓展和积累我们在金属钴氢配合物领 域里的研究工作, 本工作我们以苯硒酚为原料替代苯硫 酚制备了新型钴氢配合物, 并且研究了相应氢化物的化 学反应性质.

\section{1 结果与讨论}

\section{$1.1 \mathrm{Co}(\mathrm{II})$ 配合物 $\left[\mathrm{Co}\left(4-\mathrm{MeOC}_{6} \mathrm{H}_{4} \mathrm{Se}\right)_{2}\left(\mathrm{PMe}_{3}\right)_{3}\right]$ (1)的 制备}

将 4-甲氧基苯硒酚在 $-78{ }^{\circ} \mathrm{C}$ 的正戊烷溶液中与 $\left[\mathrm{Co}\left(\mathrm{PMe}_{3}\right)_{4}\right]$ 反应, 溶液的颜色立即由黄色转化为红棕 色, 同时析出大量红棕色粉末. 用乙醚萃取红棕色粉末, 过滤后在一 $20{ }^{\circ} \mathrm{C}$ 下由乙醚溶液中析出暗红色晶体, 为 $\mathrm{Co}(\mathrm{II})$ 配合物 1 (Eq. 1), 产率为 $51 \%$.

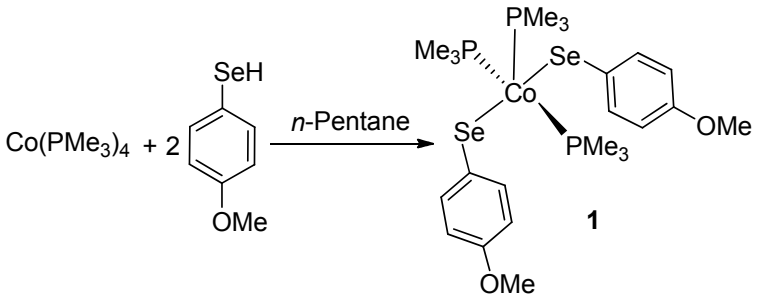

配合物 1 的分子结构由单晶 $X$ 射线衍射分析所证实 (图 1). 钴原子处于一个变形的四方锥 $(\tau=0.08)$ 的四方 面中心, $\mathrm{P}(2)$ 原子则占据该四方锥的顶点位置. 由于键 角 $\mathrm{P}(1)-\mathrm{Co}(1)-\mathrm{P}(3)\left[167.10(9)^{\circ}\right]$ 和 $\mathrm{Se}(1)-\mathrm{Co}(1)-\mathrm{Se}(2)$ $\left[162.14(6)^{\circ}\right]$ 偏离了理想值 $\left(180^{\circ}\right)$, 因此, 键角 $\mathrm{P}(2)-$ $\mathrm{Co}(1)-\mathrm{P}(3)\left[96.65(9)^{\circ}\right], \mathrm{P}(2)-\mathrm{Co}(1)-\mathrm{P}(1)\left[95.85(9)^{\circ}\right]$, $\mathrm{P}(2)-\mathrm{Co}(1)-\mathrm{Se}(1)\left[98.12(7)^{\circ}\right]$ 和 $\mathrm{P}(2)-\mathrm{Co}(1)-\mathrm{Se}(2)$ $\left[99.68(7)^{\circ}\right]$ 也偏离了理想值 $\left(90^{\circ}\right)$. 四方面上四个配位键 角 $\left(\mathrm{P}(1)-\mathrm{Co}(1)-\mathrm{Se}(1)\left[88.63(7)^{\circ}\right], \mathrm{P}(1)-\mathrm{Co}(1)-\mathrm{Se}(2)\right.$ $\left[88.17(7)^{\circ}\right], \mathrm{P}(3)-\mathrm{Co}(1)-\mathrm{Se}(1)\left[86.49(7)^{\circ}\right]$ 和 $\mathrm{P}(3)-$ $\mathrm{Co}(1)-\mathrm{Se}(2)\left[92.83(7)^{\circ}\right]$ 之和为 $356.12^{\circ}$. 这说明五个原 子 $[\mathrm{Co}(1) \mathrm{P}(1) \mathrm{P}(3) \mathrm{Se}(1) \mathrm{Se}(2)]$ 的共平面性不好. 两个 4- $\mathrm{MeOC}_{6} \mathrm{H}_{4}$ 基团是向顶点配位键 $\mathrm{Co}(1)-\mathrm{P}(2)$ 的反方向 伸展的, 显然, 这样可以使基团之间的排斥力最小, 能 量最低.

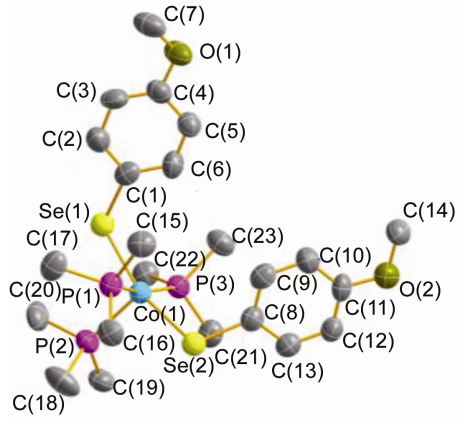

图 1 配合物 1 的分子结构

Figure 1 Molecular structure of complex 1

Selected bond lengths $(\AA)$ and bond angles $\left(^{\circ}\right)$ : $\mathrm{Co}(1)-\operatorname{Se}(1)$ 2.3907(14), $\mathrm{Co}(1)-\mathrm{Se}(2)$ 2.3745(14), $\mathrm{Co}(1)-\mathrm{P}(1)$ 2.224(2), $\mathrm{Co}(1)-\mathrm{P}(2)$ 2.252(2), $\mathrm{Co}(1)-\mathrm{P}(3)$ 2.233(2); $\mathrm{P}(1)-\mathrm{Co}(1)-\mathrm{P}(3)$ 167.11(7), $\mathrm{Se}(1)-\mathrm{Co}(1)-$ $\mathrm{Se}(2)$ 162.15(6), $\mathrm{P}(2)-\mathrm{Co}(1)-\mathrm{Se}(1)$ 98.13(7), $\mathrm{P}(2)-\mathrm{Co}(1)-\mathrm{Se}(2)$ 99.67(7)

我们推测配合物 1 的可能生成机理如 Scheme 1 所 示. 在解离一分子三甲基膦配体的前提下，4-甲氧基苯 硒酚在钴中心发生氧化加成反应生成 $\operatorname{Co}(\mathrm{II})$ 氢化物中间 体 1A. 1A 不稳定，通过单分子还原消除转化为中间物 $1 \mathrm{~B}$ ，同时释放出氢气. $\mathrm{Co}(\mathrm{I})$ 配合物 $1 \mathrm{~B}$ 与第二个分子的 4-甲氧基苯硒酚发生氧化加成反应生成不稳定的 Co(III) 氢化物中间体 1C. 1C 通过释放氢气的单分子还原消除 转化为最终产物 1 . 配合物 1 具有成单电子是顺磁性的. 但是实验中没有分离到中间体 $\mathbf{1 A} \sim 1 \mathrm{C}$.

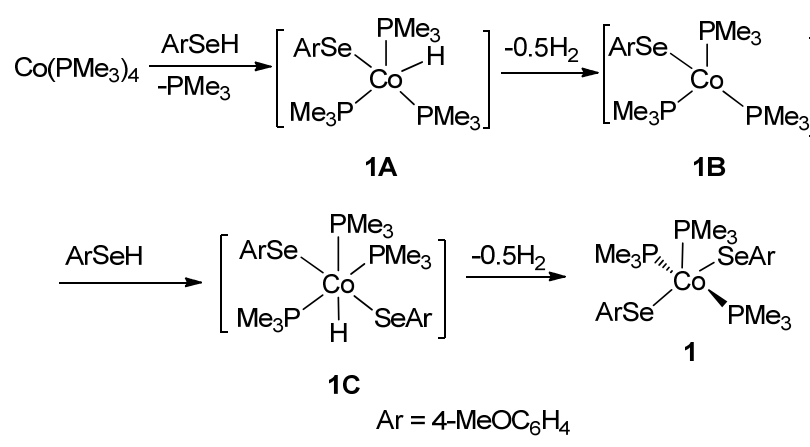

图式 1 配合物 1 形成的可能机理

Scheme 1 Proposed mechanism of formation of complex 1

\section{2 双核钴配合物 $\left[\mathrm{Co}\left(\mathrm{PMe}_{3}\right)_{2}\left(\mathrm{SeC}_{6} \mathrm{H}_{4}-2-\mathrm{Me}\right)\right]_{2}$ (2)的} 制备

相同反应条件下，当选择 2 -甲基苯硒酚与 $\left[\mathrm{Co}\left(\mathrm{PMe}_{3}\right)_{4}\right]$ 反应时，既没有得到钴氢配合物，也没有得 到配合物 1 的类似物，而是得到了双核 $\mathrm{Co}(\mathrm{I})$ 配合物 2 (Eq. 2), 产率为 $46 \%$. 当我们重新回顾我们早期的工作, 以硫酚为原料与 $\left[\mathrm{CoMe}\left(\mathrm{PMe}_{3}\right)_{4}\right]$ 反应时分离得到的产物 之一就是这类双核 $\mathrm{Co}(\mathrm{I})$ 配合物 $^{[42]} \cdot \mathrm{Co}(\mathrm{I})$ 配合物 2 是顺磁 性物质，其组成经过元素分析证实，配合物 2 的结构应 该与其硫配位的类似物相似 ${ }^{[42]}$. 


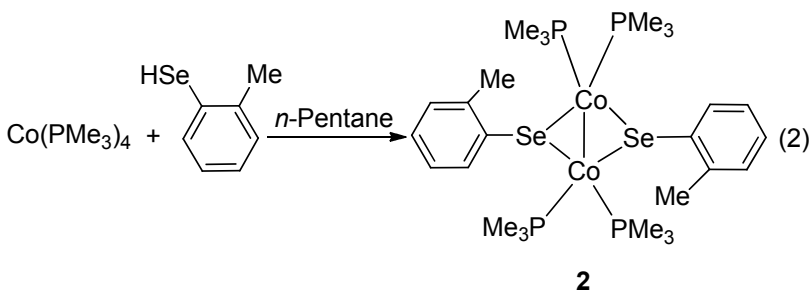

为了进一步证实配合物 $\mathbf{2}$ 的结构, 我们设计了配合 物 2 和 $\mathrm{CO}$ 的反应. 在 $0.1 \mathrm{MPa}$ 的 $\mathrm{CO}$ 气压下, 配合物 $\mathbf{2}$ 在正戊烷的溶液中逐渐转化为双羰基配合物 3 (Eq. 3). 这一结果与我们早期的工作完全一致 ${ }^{[42]}$. 配合物 3 为黄 色晶体, 在空气中十分稳定.

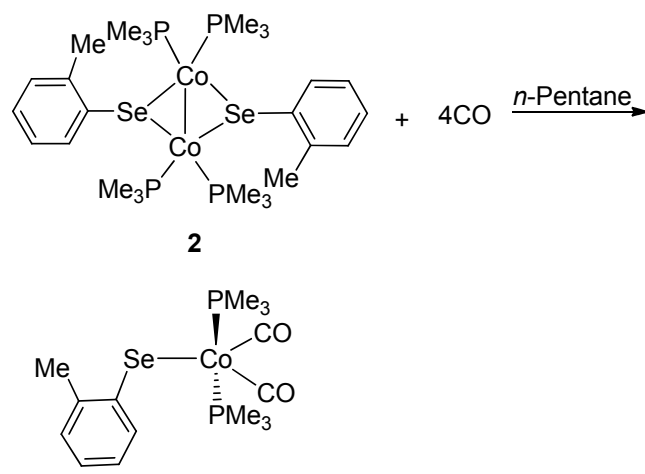

3

在配合物 3 的红外光谱中, 两个端崖基强的红外吸 收谱带分别处于 1968 和 $1893 \mathrm{~cm}^{-1}$, 该数值与其硫配位 的类似物十分相近 ${ }^{[42]}$. 单晶 $\mathrm{X}$ 射线衍射分析证实配合物 3 具有以钴为中心的三角双雉结构(图 2). 两个三甲基磷 配体处于轴向的位置. 键角 $\mathrm{P}(1)-\mathrm{Co}(1)-\mathrm{P}(2)$ 为 $175.31(2)^{\circ}$, 稍微偏离理想值 $180^{\circ}$. 在赤道平面上, 由于 苯环向 $\mathrm{C}(1)-\mathrm{O}(1)$ 键的方向伸展 $[\mathrm{Co}(1)-\mathrm{Se}(1)-\mathrm{C}(3)=$ 113.09(5) ${ }^{\circ}$, 使得键角 $\operatorname{Se}(1)-\mathrm{Co}(1)-\mathrm{C}(1)\left[129.94(6)^{\circ}\right]$ 显著地大于键角 $\mathrm{Se}(1)-\mathrm{Co}(1)-\mathrm{C}(2)\left[104.77(6)^{\circ}\right]$. 因此,

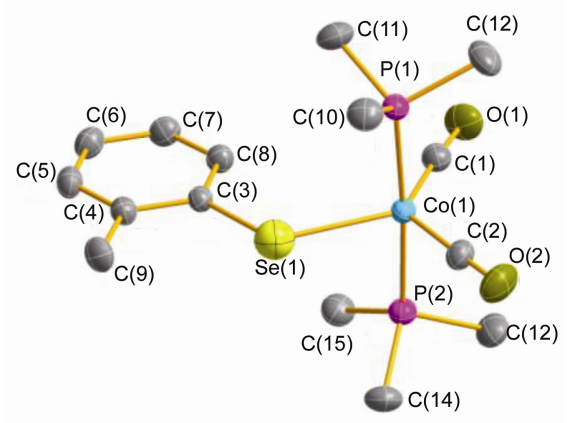

图 2 配合物 3 的分子结构

Figure 2 Molecular structure of complex 3 Selected bond lengths $(\AA)$ and bond angles $\left({ }^{\circ}\right)$ : $\mathrm{Co}-\mathrm{Se}(1) 2.4384(3)$, $\mathrm{Co}(1)-\mathrm{P}(1)$ 2.1909(5), $\mathrm{Co}(1)-\mathrm{P}(2)$ 2.1933(5), $\mathrm{Co}(1)-\mathrm{C}(1)$ 1.7417(17), $\mathrm{Co}(1)-\mathrm{C}(2)$ 1.7603(17); $\mathrm{P}(1)-\mathrm{Co}(1)-\mathrm{P}(2)$ 175.312(18), $\mathrm{C}(1)-$ $\mathrm{Co}(1)-\mathrm{Se}(1)$ 129.94(6), $\mathrm{C}(1)-\mathrm{Co}(1)-\mathrm{C}(2)$ 125.28(8), $\mathrm{C}(2)-\mathrm{Co}(1)-$ $\mathrm{Se}(1) 104.77(6)$
分子也失去了轴对称性. 配合物 3 只有一个处于赤道平 面的对称面. 该反应也说明, 我们对于反应 Eq. 2 的推 测是合理的.

\section{3 钴氢配合物 $\left[m e r-\mathrm{Co}(\mathrm{H})(\mathrm{SePh})_{2}\left(\mathrm{PMe}_{3}\right)_{3}\right](4)$ 和双 核钴配合物 $\left[\mathrm{Co}\left(\mathrm{PMe}_{3}\right)_{2}(\mathrm{PhSe})\right]_{2}(5)$ 的制备}

将 $2 \mathrm{~mol}$ 的苯硒酚与 $1 \mathrm{~mol}\left[\mathrm{Co}\left(\mathrm{PMe}_{3}\right)_{4}\right]$ 配合物在一 $78{ }^{\circ} \mathrm{C}$ 的乙醚溶液中混合(Eq. 4), 温度逐渐升至室温, 并 继续反应 $3 \mathrm{~h}$, 得到棕黄色悬浊液, 过滤后在 $-20{ }^{\circ} \mathrm{C}$ 下 由乙醚溶液中析出棕黄色晶体, 为 $\mathrm{Co}(\mathrm{III})$ 的氢化物 4 , 产率为 $18 \%$. 母液抽干后继续用正戊烷萃取，由滤液中 析出配合物 $\mathbf{5}$, 产率 $40 \%$.

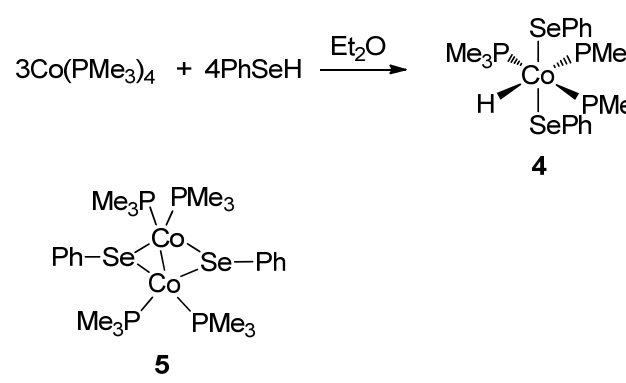

在配合物 4 的红外光谱中, 化学键 $(\mathrm{Co}-\mathrm{H})$ 的伸缩 振动出现在 $1955 \mathrm{~cm}^{-1}$ 处. 与同系列的硫配位的钴氢配 合物 $\left[v(\mathrm{Co}-\mathrm{H})=1977 \mathrm{~cm}^{-1}\right]$ 相比，这种红移是由于硒取 代配位所造成的 ${ }^{[42]}$. 在配合物 $\mathbf{4}$ 的核磁共振氢谱中, 氢 配体的质子共振信号以 $\mathrm{dt}$ 峰的形式出现在 $\delta-9.90$, 相 应的偶合常数分别为 ${ }^{2} J_{\mathrm{PH}}=103$ 和 $71 \mathrm{~Hz}$. 在配合物 4 的 核磁共振磷谱中，两组不同的共振磷信号以 $2: 1$ 的积 分比例分别出现在 $\delta-1.20$ 和 -7.55 . 这说明三个配位 的 $\mathrm{PMe}_{3}$ 配体具有两种不同的化学环境, 符合子午线排 列(meridional)方式.

单晶的 X 射线衍射分析进一步证实配合物 4 的结 构. 配合物 4 具有六配位的变形八面体结构(图 3). 三个 配位的三甲基膦配体属于典型的子午线式排列. 如果我 们指认 $\operatorname{Se}(2)-\operatorname{Co}(1)-\operatorname{Se}(1)\left[176.73(4)^{\circ}\right]$ 为轴向方向, 则 中心钴原子则处于 $[\mathrm{HP}(1) \mathrm{P}(2) \mathrm{P}(3)]$ 四原子所确定的赤道 平面中. 键角 $\mathrm{Co}(1)-\mathrm{Se}(1)-\mathrm{C}(1)$ 和 $\mathrm{Co}(1)-\mathrm{Se}(1)-\mathrm{C}(7)$ 分别为 114.6(2) 和 113.3(2) . 由于氢配体的小体积使得 两个苯环的伸展方向均为 $\mathrm{Co}-\mathrm{H}$ 键的方向, 这样会使 配合物 4 的结构最稳定，能量最低. 与键长 $\mathrm{Co}(1)-\mathrm{P}(1)$ [2.221(2) $\AA]$ 和 $\operatorname{Co}(1)-\mathrm{P}(3)$ [2.245(2) $\AA]$ 相比, 键长 $\mathrm{Co}(1)-\mathrm{P}(2)(2.270(2) \AA)$ 更长一点, 这可归因于负氢配 体强的反位影响所带来的结果. 我们早期的研究也证实 了这个结论 ${ }^{[42]} . \mathrm{Co}(\mathrm{III})$ 的氢化物 4 固体状态下在空气中 相对稳定，可以稳定存在大约 $2 \mathrm{~h}$, 但在溶液中非常不 稳定，遇到空气立即分解. 而且其溶液在室温下即使是 氮气保护下也会逐渐分解，这或许是其产率较低的原 
因. 配合物 5 的组成经过元素分析表征初步证实, 5 应该 具有与 2 类似的结构.

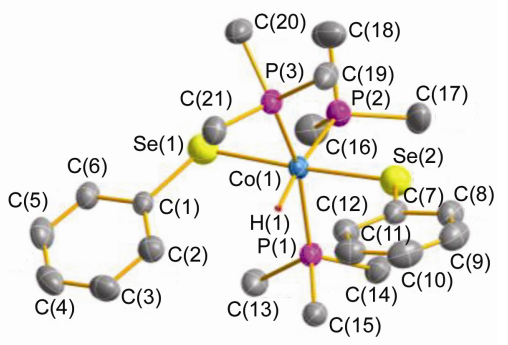

图 3 配合物 4 的分子结构

Figure 3 Molecular structure of complex 4

Selected bond lengths $(\AA)$ and bond angles $\left({ }^{\circ}\right)$ : $\mathrm{Co}(1)-\mathrm{H}(1)$ 1.31(4), $\mathrm{Co}(1)-\mathrm{Se}(1)$ 2.4015(8), $\mathrm{Co}(1)-\mathrm{Se}(2)$ 2.4036(8), $\mathrm{Co}(1)-\mathrm{P}(1)$ 2.2210(15), $\mathrm{Co}(1)-\mathrm{P}(2)$ 2.2695(17), $\mathrm{Co}(1)-\mathrm{P}(3)$ 2.2453(15); $\mathrm{Se}(1)-$ $\mathrm{Co}(1)-\mathrm{Se}(2)$ 176.73(4), $\mathrm{P}(1)-\mathrm{Co}(1)-\mathrm{Se}(1)$ 95.80(4), $\mathrm{P}(1)-\mathrm{Co}(1)-$ $\mathrm{Se}(2)$ 86.36(4), $\mathrm{P}(1)-\mathrm{Co}(1)-\mathrm{P}(2)$ 97.13(6), $\mathrm{P}(1)-\mathrm{Co}(1)-\mathrm{P}(3)$ 158.18(7), $\mathrm{P}(2)-\mathrm{Co}(1)-\mathrm{Se}(1)$ 88.15(5), $\mathrm{P}(2)-\mathrm{Co}(1)-\mathrm{Se}(2)$ 89.14(5), $\mathrm{P}(3)-\mathrm{Co}(1)-\mathrm{Se}(1)$ 85.00(4), $\mathrm{P}(3)-\mathrm{Co}(1)-\mathrm{Se}(2)$ 93.91(4), $\mathrm{P}(3)-$ $\mathrm{Co}(1)-\mathrm{P}(2) 104.70(6)$

\section{4 钴氢配合物 4 的催化性能探索}

为了加深对钴氢配合物性质的认识, 我们初步探索 了钴氢配合物 4 在醛和酮硅氢化反应中的催化性能. 在 $5 \mathrm{~mol} \% 4$ 存在下, 以三乙氧基硅烷为氢源, 苯甲醛在 $50{ }^{\circ} \mathrm{C}$ 下反应 $4 \mathrm{~h}$, 即可通过薄层色谱(TLC)检测到硅醚 产物的存在. 遗憾的是, 即使继续反应 $24 \mathrm{~h}$ 仍未能转化 完全. 升高温度到 $60{ }^{\circ} \mathrm{C}$ 继续反应 $12 \mathrm{~h}$ 仍没有明显变化. 我们对底物进行了拓展, 结果见表 1. 实验结果表明, 以 $5 \mathrm{~mol} \% 4$ 作为催化剂, 三乙氧基硅烷作为氢源, 在 $50{ }^{\circ} \mathrm{C}$ 的条件下, 钴氢配合物对醛的硅氢化反应有一定 的催化效果, 但与我们之前报道的钴氢化合物的催化效 果相比 ${ }^{[39,40]}$ 转化率较低, 而对酮的催化效果更差. 我们 推测催化效果不理想的原因是由钴氢配合物 $\mathbf{4}$ 的不稳定 性造成的.

推测配合物 4 催化醛酮硅氢化反应的可能机理如 Scheme 2 所示. 首先, 一分子羰基化合物取代一分子三 甲基膦配体生成 $\pi(\mathrm{C}=\mathrm{O})$ 配位的中间体 a. 然后, 氢配 体亲核进攻羰基上的碳原子生成中间体 $\mathbf{b}$, 同时产生一 个配位空缺. 三乙氧基硅烷占据中间体 $\mathbf{b}$ 上的配位空穴 形成中间体 c. 最后, 在三甲基膦存在下通过硅氢键活 化消除一分子硅醚，同时使催化剂 4 再生.

\section{2 结论}

利用苯硒酚和 $\left[\mathrm{Co}\left(\mathrm{PMe}_{3}\right)_{4}\right]$ 的反应制得了 $\mathrm{Co}(\mathrm{III})$ 氢 配合物 $\left[m e r-\mathrm{Co}(\mathrm{H})(\mathrm{SePh})_{2}\left(\mathrm{PMe}_{3}\right)_{3}\right]$ (4). 4-甲氧基苯硒酚 与 $\left[\mathrm{Co}\left(\mathrm{PMe}_{3}\right)_{4}\right]$ 反应则生成了 $\mathrm{Co}(\mathrm{II})$ 配合物 $[\mathrm{Co}(4-\mathrm{MeO}-$ $\left.\left.\mathrm{C}_{6} \mathrm{H}_{4} \mathrm{Se}\right)_{2}\left(\mathrm{PMe}_{3}\right)_{3}\right]$ (1). 而 2-甲基苯硒酚与 $\left[\mathrm{Co}\left(\mathrm{PMe}_{3}\right)_{4}\right]$ 反 应生成了双核钴配合物 $\left[\mathrm{Co}\left(\mathrm{SeC}_{6} \mathrm{H}_{4}-2-\mathrm{Me}\right)\left(\mathrm{PMe}_{3}\right)_{2}\right]_{2}$ (2).
表 1 配合物 4 对醛酮硅氢化反应的催化性质研究 ${ }^{a}$

Table 1 Catalytic property of complex 4 in hydrosilylation of aldehydes and ketones

Entry Substrate $\mathrm{Conv}^{b} \% \mathrm{Yield}^{c} \% \%$

${ }^{a}$ Reaction conditions: $\mathrm{RCHO}$ or acetophenone $(1.0 \mathrm{mmol}),(\mathrm{EtO})_{3} \mathrm{SiH}(1.2$ $\mathrm{mmol})$, and $4(0.05 \mathrm{mmol})$ in $3 \mathrm{~mL}$ of THF; $50{ }^{\circ} \mathrm{C}, 24 \mathrm{~h} .{ }^{b}$ Determined by GC with $n$-dodecane as an internal standard. ${ }^{c}$ The isolated yield is the average of the four parrallel experiments.

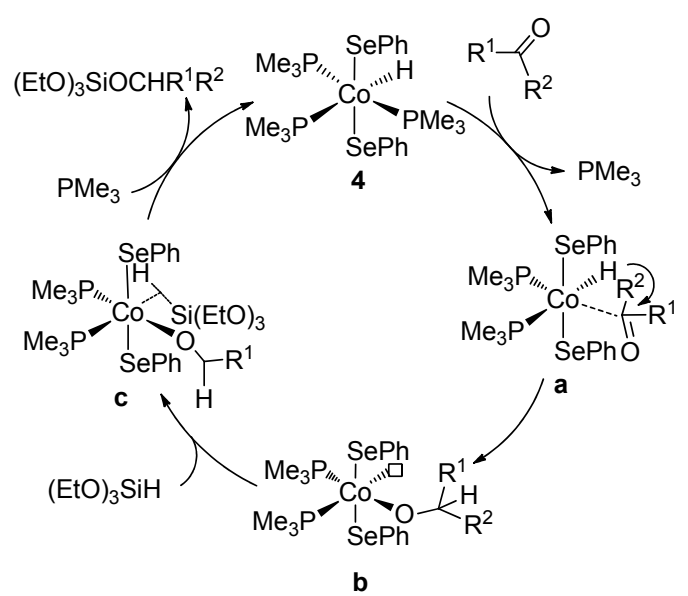

图式 2 配合物 4 催化硅氢化反应的可能机理

Scheme 2 Proposed mechanism of hydrosilylation catalyzed by complex 4

初步探索了钴氢配合物 $\mathbf{4}$ 在醛和酮硅氢化反应中的催化 性能. 结果表明，以 $5 \mathrm{~mol} \%$ 的 4 作为催化剂，三乙氧基 硅烷为氢源, 在 $50{ }^{\circ} \mathrm{C}$ 的条件下, 钴氢配合物对醛的硅 氢化反应有一定的催化效果, 而对酮的催化效果较差. 这或许是由钴氢配合物 $\mathbf{4}$ 的不稳定性造成的. 双核钴配 合物 2 和 $\mathrm{CO}$ 进一步反应，生成双羰基钴配合物 $[\mathrm{Co}(\mathrm{Se}-$ 
$\left.\left.\mathrm{C}_{6} \mathrm{H}_{4}-2-\mathrm{Me}\right)(\mathrm{CO})_{2}\left(\mathrm{PMe}_{3}\right)_{2}\right](3)$. 上述实验结果说明, 各种 苯硒酚的衍生物与 $\left[\mathrm{Co}\left(\mathrm{PMe}_{3}\right)_{4}\right]$ 反应的产物是各具特色 的. 之所以会产生这种结果, 我们推测取代基在此起了 很重要的作用. 4-甲氧基苯硒酚由于含有强给电子取代 基致使钴氢中间体非常不稳定, 会迅速生成溶解度更差 的配合物 1. 2-甲基苯硒酚由于邻位含有给电子取代基 致使空间位阻变大, 一方面阻碍了进一步结合邻甲基苯 硒酚产生钴氢配合物，再加上给电子基导致钴氢配合物 稳定性变差, 从而使配合物 2 成为主要产物. 到目前为 止, 由于我们选择的苯硒酚种类有限, 还没有找到该反 应的规律性. 在该研究领域, 还有许多研究工作需要进 一步地探索.

\section{3 实验部分}

\section{1 仪器与试剂}

核磁共振谱 ${ }^{1} \mathrm{H}$ NMR, ${ }^{31} \mathrm{P}$ NMR 和 ${ }^{13} \mathrm{C}$ NMR 在 Bruker AV 300 (300 MHz)核磁共振仪上测定; 元素分析 则使用了 Elementer Vario ELIII 进行测量; 化合物的熔 点用上海申光 WRS-1C 型熔点仪测定, 温度未经校正; 质谱则是在 Agilent Technologies 6510 QTOF LC/MS 型 质谱仪中测出; 红外图谱用 Bruker ALPHA FI-IR 型测试 仪测定.

所有溶剂均为分析纯级. 乙醚、正戊烷、四氢呋喃 使用前均在氮气氛中经钠-二苯甲酮回流处理. 试剂邻 溴甲苯、对溴苯甲醚从潍坊鸣舟化工有限公司购买, 溴 苯购自国药集团化学试剂有限公司, 醛酮底物从上海海 曲化工有限公司购买, 正十二烷购自天津科密欧化学试 剂有限公司, 乙醚、四氢呋喃从天津富宇精细化工有限 公司购买, 正戊烷购自天津市大茂化学试剂厂; $\mathrm{PhSeH}$, $o-\mathrm{MeC}_{6} \mathrm{H}_{4} \mathrm{SeH}, p-\mathrm{MeOC}_{6} \mathrm{H}_{4} \mathrm{SeH}$ 和 $\left[\mathrm{Co}\left(\mathrm{PMe}_{3}\right)_{4}\right]$ 根据文献 [43, 44]方法合成.

除非特别说明, 所有试验均采用 Schlenck 实验技 术, 在氮气保护的无水无氧条件下进行. 实验中涉及到 的 $\mathrm{CO}$ 为高纯气体.

\section{2 实验方法}

\subsection{1 $\left[\mathrm{Co}\left(4-\mathrm{MeOC}_{6} \mathrm{H}_{4} \mathrm{Se}\right)_{2}\left(\mathrm{PMe}_{3}\right)_{3}\right]$ (1) 的合成}

将 $p$ - $\mathrm{MeOC}_{6} \mathrm{H}_{4} \mathrm{SeH}(0.57 \mathrm{~g}, 3.0 \mathrm{mmol})$ 溶于 $30 \mathrm{~mL}$ 正 戊烷溶液中, 在 $-78{ }^{\circ} \mathrm{C}$ 的条件下, 将其加入到 $30 \mathrm{~mL}$ $\mathrm{Co}\left(\mathrm{PMe}_{3}\right)_{4}(0.55 \mathrm{~g}, 1.5 \mathrm{mmol})$ 的正戊烷溶液中. 反应迅 速产生大量红棕色沉淀, 恢复室温后, 继续反应 $3 \mathrm{~h}$. 溶 液经过滤后得到红棕色固体. 将红棕色固体在乙醚溶液 中于 $-20{ }^{\circ} \mathrm{C}$ 重结晶. 配合物 1 以红棕色晶体的形式在 乙醚溶液中析出, 产量 $0.51 \mathrm{~g}(51 \%)$. m.p. $>200{ }^{\circ} \mathrm{C}$ (dec.); IR (Nujol) v: $1586(\mathrm{C}=\mathrm{C}), 944\left(\mathrm{PMe}_{3}\right) \mathrm{cm}^{-1}$. Anal. calcd for $\mathrm{C}_{23} \mathrm{H}_{41} \mathrm{CoO}_{2} \mathrm{P}_{3} \mathrm{Se}_{2}$ : C 41.90, H 6.27; found $\mathrm{C}$
41.52, H 6.09 .

\subsection{2 $\left[\mathrm{Co}\left(\mathrm{PMe}_{3}\right)_{2}\left(\mathrm{SeC}_{6} \mathrm{H}_{4}-2-\mathrm{Me}\right)\right]_{2}$ (2) 的合成}

将 $o-\mathrm{MeC}_{6} \mathrm{H}_{4} \mathrm{SeH}(0.55 \mathrm{~g}, 3.2 \mathrm{mmol})$ 溶于 $30 \mathrm{~mL}$ 正戊 烷溶液中, 在 $-78{ }^{\circ} \mathrm{C}$ 的条件下, 将其加入到 $30 \mathrm{~mL}$ $\mathrm{Co}\left(\mathrm{PMe}_{3}\right)_{4}(1.16 \mathrm{~g}, 3.2 \mathrm{mmol})$ 的正戊烷溶液中. 室温下 搅拌反应 $3 \mathrm{~h}$, 得到红棕色悬浊液. 溶液经过滤后置于 $-20{ }^{\circ} \mathrm{C}$ 下, 配合物 2 以深红色晶体的形式在正戊烷溶 液中析出, 产量 $0.56 \mathrm{~g}$ (46\%). m.p. $>74{ }^{\circ} \mathrm{C}$ (dec.); IR (Nujol) v: $1584(\mathrm{C}=\mathrm{C}), 944\left(\mathrm{PMe}_{3}\right) \mathrm{cm}^{-1}$. Anal. calcd for $\mathrm{C}_{26} \mathrm{H}_{50} \mathrm{Co}_{2} \mathrm{P}_{4} \mathrm{Se}_{2}$ : C 40.96, H 6.61; found C 41.29, H 6.50.

\subsection{3 $\left[\mathrm{Co}\left(\mathrm{PMe}_{3}\right)_{2}(\mathrm{CO})_{2}\left(\mathrm{SeC}_{6} \mathrm{H}_{4}-2-\mathrm{Me}\right)\right](3)$ 的合成}

室温下, 将配合物 $2(0.77 \mathrm{~g}, 1.00 \mathrm{mmol})$ 溶于 $60 \mathrm{~mL}$ 正戊烷中, 充入一个大气压的 $\mathrm{CO}$ 气体, 溶液颜色由红 棕色变成黄色. 继续摚拌反应 $36 \mathrm{~h}$, 溶液经过滤后置于 $4{ }^{\circ} \mathrm{C}$. 配合物 3 以黄色晶体的形式从正戊烷溶液中析出, 产量为 0.59 g (67\%). m.p. $>125{ }^{\circ} \mathrm{C}$ (dec.); ${ }^{1} \mathrm{H}$ NMR (300 $\left.\mathrm{MHz}, \mathrm{C}_{6} \mathrm{D}_{6}\right) \delta: 1.32$ (s,18H, PMe3), 2.94 (s, 3H, CH3), 7.13 (d, $J=6.9 \mathrm{~Hz}, 3 \mathrm{H}, \mathrm{CH}), 7.87$ (d, $J=7.2 \mathrm{~Hz}, 1 \mathrm{H}, \mathrm{CH}$ ); ${ }^{31} \mathrm{P}$ NMR $\left(121.5 \mathrm{MHz}, \mathrm{C}_{6} \mathrm{D}_{6}\right) \delta: 35.32 ;{ }^{13} \mathrm{C}$ NMR $(75 \mathrm{MHz}$, $\left.\mathrm{C}_{6} \mathrm{D}_{6}, 298 \mathrm{~K}\right) \delta: 18.71\left(\mathrm{t}, J=17.7 \mathrm{~Hz}, \mathrm{PMe}_{3}\right), 24.42(\mathrm{~s}$, $\mathrm{Me}), 122.63,125.57,128.94,132.28,138.63,140.76$, 202.03 (s, CO); IR (Nujol) v: 1968, 1893 (CO), 1580 (C= C), $938\left(\mathrm{PMe}_{3}\right) \mathrm{cm}^{-1}$. Anal. calcd for $\mathrm{C}_{15} \mathrm{H}_{27} \mathrm{CoO}_{2} \mathrm{P}_{2} \mathrm{Se}: \mathrm{C}$ 41.21, H 6.22; found C 41.47, H 5.89.

3.2.4 $\left[\right.$ mer $\left.-\mathrm{Co}(\mathrm{H})(\mathrm{SePh})_{2}\left(\mathrm{PMe}_{3}\right)_{3}\right]$ (4) 和 $\left[\mathrm{Co}\left(\mathrm{PMe}_{3}\right)_{2}-\right.$ $(\mathrm{PhSe})]_{2}(5)$ 的合成

将 $\mathrm{PhSeH}(0.60 \mathrm{~g}, 3.8 \mathrm{mmol})$ 溶于 $30 \mathrm{~mL}$ 乙醚溶液中, 在 $-78{ }^{\circ} \mathrm{C}$ 的条件下, 将其加入到 $30 \mathrm{~mL} \mathrm{Co}\left(\mathrm{PMe}_{3}\right)_{4}$ $(0.69 \mathrm{~g}, 1.9 \mathrm{mmol})$ 的乙醚溶液中. 室温下搅拌反应 $3 \mathrm{~h}$, 得到棕黄色悬浊液。溶液经过滤后置于 $-20{ }^{\circ} \mathrm{C}$. 配合 物 4 以棕黄色晶体的形式在乙醚溶液中析出, 产量为 $0.21 \mathrm{~g}(18 \%)$. 母液在真空下转干溶剂后继续用 $30 \mathrm{~mL}$ 正戊烷萃取, 过滤后置于 $-20{ }^{\circ} \mathrm{C}$. 配合物 $\mathbf{5}$ 以深红色晶 体的形式在正戊烷溶液中析出, 产量为 $0.28 \mathrm{~g}(40 \%)$.

4: m.p. $81 \sim 83{ }^{\circ} \mathrm{C}$ (dec.); ${ }^{1} \mathrm{H}$ NMR $\left(300 \mathrm{MHz}, \mathrm{C}_{6} \mathrm{D}_{6}\right)$ $\delta:-9.90\left(\mathrm{dt},{ }^{2} J_{\mathrm{PtrannH}}=103 \mathrm{~Hz},{ }^{2} J_{\mathrm{P} c i s \mathrm{H}}=71 \mathrm{~Hz}, 1 \mathrm{H}\right.$, Co-H), $1.22\left(\mathrm{~d},{ }^{2} J_{\mathrm{PH}}=6.9 \mathrm{~Hz}, 9 \mathrm{H}, \mathrm{PMe}_{3}\right), 1.48\left(\mathrm{t},\left.\right|^{2} J_{\mathrm{PH}}+\right.$ $\left.{ }^{4} J_{\mathrm{PH}} \mid=3.6 \mathrm{~Hz}, 18 \mathrm{H}, \mathrm{PMe}_{3}\right), 7.11\left(\mathrm{t},{ }^{3} J=7.2 \mathrm{~Hz}, 2 \mathrm{H}, \mathrm{CH}\right)$, 7.20 (t, $\left.{ }^{3} J=7.8 \mathrm{~Hz}, 4 \mathrm{H}, \mathrm{CH}\right), 8.44\left(\mathrm{~d},{ }^{3} J=7.5 \mathrm{~Hz}, 4 \mathrm{H}\right.$, $\mathrm{CH}) ;{ }^{31} \mathrm{P}$ NMR $\left(121.5 \mathrm{MHz}, \mathrm{C}_{6} \mathrm{D}_{6}\right) \delta:-7.55(\mathrm{~s}, 1 \mathrm{P}$, $\left.\mathrm{PMe}_{3}\right),-1.20$ (s, 2P, $\left.\mathrm{PMe}_{3}\right) ;{ }^{13} \mathrm{C} \mathrm{NMR}\left(75 \mathrm{MHz}, \mathrm{C}_{6} \mathrm{D}_{6}\right) \delta$ : $17.39\left(\mathrm{~d},{ }^{2} J_{(\mathrm{PH})}=21.8 \mathrm{~Hz}, \mathrm{PMe}_{3}\right), 20.93\left(\mathrm{t},\left.\right|^{2} J_{(\mathrm{PH})}+{ }^{4} J_{(\mathrm{PH})} \mid=\right.$ $\left.15.0 \mathrm{~Hz}, \mathrm{PMe}_{3}\right), 123.07,127.93,132.99,137.24,137.42$; IR (Nujol) v: $1955(\mathrm{Co}-\mathrm{H}), 1571(\mathrm{C}=\mathrm{C}), 938\left(\mathrm{PMe}_{3}\right)$ $\mathrm{cm}^{-1}$. Anal. calcd for $\mathrm{C}_{21} \mathrm{H}_{38} \mathrm{CoP}_{3} \mathrm{Se}_{2}$ : C 42.01, H 6.38; 
found C 41.67, H 6.63.

5: m.p. $>71{ }^{\circ} \mathrm{C}$ (dec.); IR (Nujol) v: $1572(\mathrm{C}=\mathrm{C})$, $934\left(\mathrm{PMe}_{3}\right) \mathrm{cm}^{-1}$. Anal. calcd for $\mathrm{C}_{24} \mathrm{H}_{46} \mathrm{Co}_{2} \mathrm{P}_{4} \mathrm{Se}_{2}$ : C 39.26, H 6.31; found C 38.86, H 6.11.

\subsection{7 催化醛酮硅氢化反应步骤}

向含有 $3 \mathrm{~mL}$ THF 的 Schlenck 管中依次加入 $1 \mathrm{mmol}$ 的醛或酮底物, $1.2 \mathrm{mmol}$ 的三乙氧基硅烷以及 0.05 $\mathrm{mmol}$ 的钴氢配合物 4 , 随后将上述体系置于 $50{ }^{\circ} \mathrm{C}$ 下加 热搅拌, 并利用 TLC 进行检测. 反应 $24 \mathrm{~h}$ 后, 向反应体 系中加入 $1 \mathrm{~mL}$ 的 $\mathrm{MeOH}$ 以及 $5 \mathrm{~mL}$ 的 $10 \%$ 的 $\mathrm{NaOH}$ 水 溶液. 随后在 $60{ }^{\circ} \mathrm{C}$ 下, 将体系加热搅拌 $24 \mathrm{~h}$ 进行水解. 反应混合物用乙醚萃取后, 用无水 $\mathrm{Na}_{2} \mathrm{SO}_{4}$ 干燥有机相, 利用气相色谱(GC)检测转化率, 并通过柱层析分离提纯 催化产物.

\section{3 配合物晶体结构测定}

单晶的 X 射线衍射实验是在 STOE STADIVARI Cu 或者 Stoe IPDS2 衍射仪上进行的. 晶体结构和分子结 构是利用 Olex $2^{[45]}$ 和 ShelXS ${ }^{[46]}$ 程序包采用直接方法确 定的. 利用最小二乘法在 ShelXL ${ }^{[47]}$ 程序包中进行了数 据精修. CCDC-1518969 (1), 1511561 (3)和 1511997 (4) 的晶体学数据, 可以从下列地址中免费获取: CCDC, 12 Union Road, Cambridge CB2 1EZ, UK (fax: ( + 44)1223-336-033; e-mail: deposit@ccdc.cam. ac.uk).

辅助材料(Supporting Information) 配合物的核磁共 振氢谱、碳谱、磷谱以及红外谱图, 醇类产物的核磁共 振氢谱、碳谱、质谱, 晶体结构的 cif 文件等. 这些材料 可以免费从本刊网站(http://sioc-journal.cn/)上下载.

\section{Referenes}

[1] Bauer, I.; Knölker, H. J. Chem. Rev. 2015, 115, 3170.

[2] Wang, W. H.; Himeda, Y.; Muckerman, J. T.; Manbeck, G. F.; Fujita, E. Chem. Rev. 2015, 115, 12936.

[3] Eberhardt, N. A.; Guan, H. R. Chem. Rev. 2016, 116, 8373.

[4] Guo, N.; Zhu, S. F. Chin. J. Org. Chem. 2015, 35, 1383 (in Chinese). (郭娜, 朱守非, 有机化学, 2015, 35, 1383.)

[5] Yuan, Q. J.; Zhang, W. B. Chin. J. Org. Chem. 2015, 36, 274 (in Chinese).

(袁乾家，张万斌，有机化学, 2015, 36, 274.)

[6] Zhang, H.; Sun, H. J.; Li, X. Y. Chin. J. Org. Chem. 2016, 36, 2843 (in Chinese). (伅花, 孙宏建, 李晓燕, 有机化学, 2016, 36, 2843.)

[7] Gunanathan, C.; Hölscher, M.; Pan, F.; Leitner, W. J. Am. Chem. Soc. 2012, 134, 14349.

[8] Jiang, Y.; Huang, W.; Schmalle, H. W.; Blacque, O.; Fox, T.; Berke, H. Organometallics 2013, 32, 7043.

[9] Dai, N.; Shang, R.; Fu, M. C.; Fu, Y. Chin. J. Chem. 2015, 33, 405.

[10] Liang, M. T.; Xia, X. F.; Liu, X.; Li, H. X. Chin. J. Chem. 2015, 33,
578.

[11] Yang, X. ACS Catal. 2012, 2, 964.

[12] Huff, C. A.; Sanford, M. S. ACS Catal. 2013, 3, 2412.

[13] Hu, P.; Diskin-Posner, Y.; Ben-David, Y.; Milstein, D. ACS Catal. 2014, 4, 2649.

[14] Arndtsen, B. A.; Bergman, R. G. Science 1995, 270, 1970.

[15] Li, C.; Widjaja, E.; Garland, M. Organometallics 2004, 23, 4131.

[16] Filonenko, G. A.; Hensen, E. J.; Pidko, E. A. Catal. Sci. Technol. 2014, 4, 3474.

[17] McGrady, G. S.; Guilera, G. Chem. Soc. Rev. 2003, 32, 383.

[18] Conifer, C.; Gunanathan, C.; Rinesch, T.; Hölscher, M.; Leitner, W. Eur. J. Inorg. Chem. 2015, 333.

[19] Chen, H.; Schlecht, S.; Semple, T. C.; Hartwig, J. F. Science 2000, $287,1995$.

[20] Hauwert, P.; Boerleider, R.; Warsink, S.; Weigand, J. J.; Elsevier, C. J. J. Am. Chem. Soc. 2010, 132, 16900.

[21] Fan, L.; Parkin, S.; Ozerov, O. V. J. Am. Chem. Soc. 2005, 127, 16772 .

[22] Lersch, M.; Tilset, M. Chem. Rev. 2005, 105, 2471.

[23] Haenel, M. W.; Oevers, S.; Angermund, K.; Kaska, W. C.; Fan, H. J.; Hall, M. B. Angew. Chem. 2001, 113, 3708.

[24] Nielsen, M.; Alberico, E.; Baumann, W.; Drexler, H. J.; Junge, H.; Gladiali, S.; Beller, M. Nature 2013, 495, 85.

[25] Clarke, Z. E.; Maragh, P. T.; Dasgupta, T. P.; Gusev, D. G.; Lough, A. J.; Abdur-Rashid, K. Organometallics 2006, 25, 4113.

[26] Jiang, Y.; Blacque, O.; Fox, T.; Frech, C. M.; Berke, H. Organometallics 2009, 28, 5493.

[27] Guo, R.; Lough, A. J.; Morris, R. H.; Song, D. Organometallics 2004, 23, 5524.

[28] Burling, S.; Kociok-Köhn, G.; Mahon, M. F.; Whittlesey, M. K.; Williams, J. M. Organometallics 2005, 24, 5868.

[29] Jones, W. D. Inorg. Chem. 2005, 44, 4475.

[30] Fu, X.; Wayland, B. B. J. Am. Chem. Soc. 2005, 127, 16460.

[31] Li, X.; Chianese, A. R.; Vogel, T.; Crabtree, R. H. Org. Lett. 2005, $7,5437$.

[32] Ding, K.; Brennessel, W. W.; Holland, P. L. J. Am. Chem. Soc. 2009, 131, 10804.

[33] Du, J. Z.; Wang, L. B.; Xie, M. H.; Deng, L. Angew. Chem., Int. Ed. 2015, 54, 12640 .

[34] Sun, J.; Luo, L.; Luo, Y.; Deng, L. Angew. Chem., Int. Ed. 2017, 56, 2720.

[35] Scheuermann, M. L.; Semproni, S. P.; Pappas, I.; Chirik, P. J. Inorg. Chem. 2014, 53, 9463 .

[36] Tokmic, K.; Markus, C. R.; Zhu, L.; Fout, A. R. J. Am. Chem. Soc. 2016, $138,11907$.

[37] Jeletic, M. S.; Helm, M. L.; Hulley, E. B.; Mock, M. T.; Appel, A. M.; Linehan, J. C. ACS Catal. 2014, 4, 3755

[38] Yu, R. P.; Darmon, J. M.; Milsmann, C.; Margulieux, G. W.; Stieber, S. C. E.; DeBeer, S.; Chirik, P. J. J. Am. Chem. Soc. 2013, 135, 13168 .

[39] Niu, Q.; Sun, H.; Li, X.; Klein, H.-F.; Flörke, U. Organometallics 2013, 32, 5235.

[40] Zhou, H.; Sun, H.; Zhang, S.; Li, X. Organometallics 2015, 34, 1479 .

[41] Xiong, Z.; Li, X.; Zhang, S.; Shi, Y.; Sun, H. Organometallics 2016, 35, 357.

[42] Jiao, G.; Li, X.; Sun, H.; Xu, X. J. Organomet. Chem. 2007, 692, 4251.

[43] Foster, D. G. Org. Synth. 1944, 24, 89.

[44] Klein, H.-F.; Karsch, H. H. Chem. Ber. 1975, 108, 944.

[45] Dolomanov, O. V.; Bourhis, L. J.; Gildea, R. J.; Howard, J. A. K.; Puschmann, H. J. Appl. Crystallogr. 2009, 42, 339.

[46] Sheldrick, G. M. Acta Crystallogr. 2008, A64, 112.

[47] Sheldrick, G. M. Acta Crystallogr. 2015, C71, 3. 